\title{
Using cellophane tape to experience interference birefringent filters
}

D. Puerto, P. Velásquez, M. M. Sánchez-López, I. Moreno, F. Mateos

D. Puerto, P. Velásquez, M. M. Sánchez-López, I. Moreno, F. Mateos, "Using cellophane tape to experience interference birefringent filters," Proc. SPIE 9664, Ninth International Topical Meeting on Education and Training in Optics and Photonics, 966428 (24 October 2005); doi: 10.1117/12.2207773

SPIE Event: Ninth International Topical Meeting on Education and Training in Optics and Photonics, 2005, Marseille, France 


\title{
Ref ETOP088
}

\section{Using cellophane tape to experience interference birefringent filters}

\author{
D. Puerto ${ }^{1}$, P. Velásquez ${ }^{1,2}$, M. M. Sánchez-López ${ }^{2,3}$, I. Moreno $^{1}$ and F. Mateos ${ }^{1}$
}

1. Dept. Ciencia y Tecnología de Materiales, Univ. Miguel Hernández. 03202 Elche, Spain

2 Instituto de Bioingeniería, Universidad Miguel Hernández, 03202 Elche, Spain

3. Departamento de Física Aplicada, Universidad Miguel Hernández. 03202 Elche, Spain.

\section{Abstract}

In this work we propose and demonstrate a very simple method to fabricate interference multilayer birefringent filters. We employ the birefringence properties of common cellophane. Cellophane tape layers can be very easily superimposed with different orientations in order to generate different spectral responses. We experimentally demonstrate this behaviour with the aid of a portable spectrophotometer. This technique represents a simple and inexpensive way of experimentally investigating the optical properties of birefringent filters.

\section{Polarization, Birefringent filters, Cellophane tape}

\section{Keywords}

\section{Introduction}

The birefringent properties of some cellophane tapes are well known and can be used in demonstrations and easy experiments such as kaleidoscopes [1,2]. The birefringence of the cellophane can be easily verified by placing a piece of tape between two crossed polarizers and observing whether it looks transparent [3]. Because cellophane tape layers can be superimposed with specific orientations, they are an easy way to build a uniaxial optical multilayer. The transmission spectra of this kind of filters can be described using usual Jones matrix formalism [4]. Therefore, a cellophane tape filter is a very useful tool to teach polarization effects, birefringence and birefringent filters.

\section{Cellophane birefringence}

The first step in the proposed experimental demonstration is to calibrate the cellophane tape birefringence as a function of the input light wavelength. For this purpose we place the cellophane tape between parallel and crossed polarizers. The cellophane tape is placed in between with the principal axis oriented at $45^{\circ}$ with respect to the polarizers. The normalized transmission is given by [5] :

$$
i_{\text {parallel }}=\cos ^{2}(\square / 2), \quad i_{\text {crossed }}=\sin ^{2}(\square / 2),
$$

where $\square$ is the phase shift introduced by the cellophane, which is given by $\phi=(2 \pi / \lambda) \Delta n d$, being $\square n$ is the difference between the ordinary and extraordinary refractive indices, $d$ the physical thickness of the layer and $\square$ the wavelength. Equation (1) indicates that the measured spectrum will be an oscillating curve as a function of the wavelength. From these relations, the phase shift can be calculated as:

$$
\phi=2 \arctan \left(\sqrt{\frac{i_{\text {crossed }}}{i_{\text {parallel }}}}\right) .
$$

Figure 1 shows a picture of the experimental setup. We employ a portable UV-visible Stellar-Net spectrophotometer, model EPP2000, operating in the range $195 \mathrm{~nm}$ to $850 \mathrm{~nm}$, a nonpolarized light from a tungsten lamp (Stellar Net SL) and two photographic linear polarizers from Hana. 


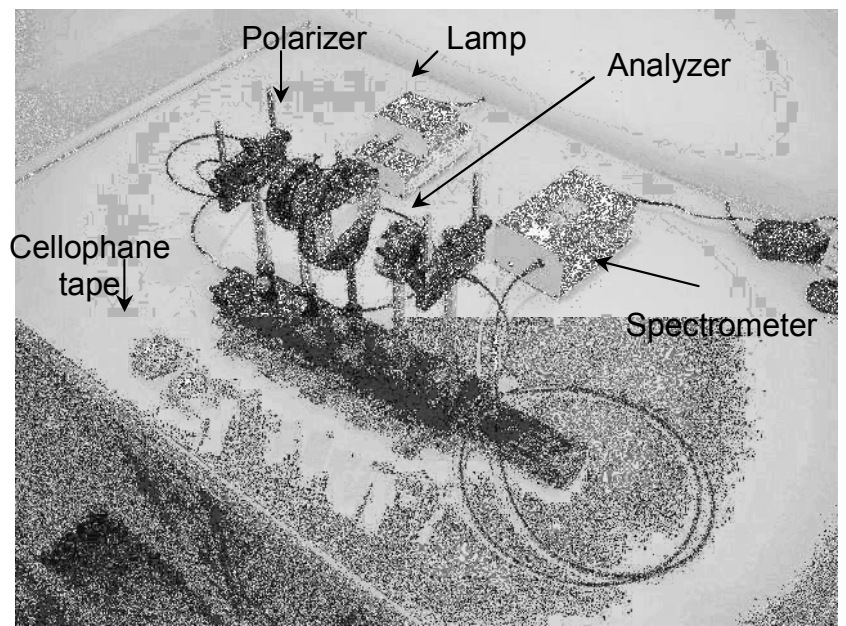

Fig. 1. Experimental setup

Figure 2 shows the measured experimental intensity transmission for parallel and crossed polarizers for one and eight cellophane layers aligned parallel. The results show the expected oscillating behaviour. The number of oscillations increases with the number of cellophane tape layers. From these experimental data, we have made a Cauchytype fit of the phase shift $\square$ as a function of the wavelength $\square[5]$.

\section{Cellophane birefringent filters}

Once the birefringence has been calibrated, the cellophane tape can be used to build birefrinfent filters. The first example we show here is a classical folded Solc filter. It consists of a set of identical wave plates whose azimuthal angle maintains its absolute value, but changes sign from one layer to the next, placed in between crossed polarizers [4]. The transmission is maximum at the wavelengths for which the birefringence is an odd multiple of $\square$, while it decreases rapidly for other wavelengths. We built a Solc filter consisting on 6 wave plates, each one composed of three parallel aligned cellophane layers, being the azimuthal angle for each consecutive wave plate $\square= \pm 7.5^{\circ}$. Figure $3(\mathrm{a})$ shows the simulated normalized transmission, and the experimental measured spectrum. The filter provides a maximum transmission centred at $\square=659 \mathrm{~nm}$, with a spectral width $\square \square=80 \mathrm{~nm}$.
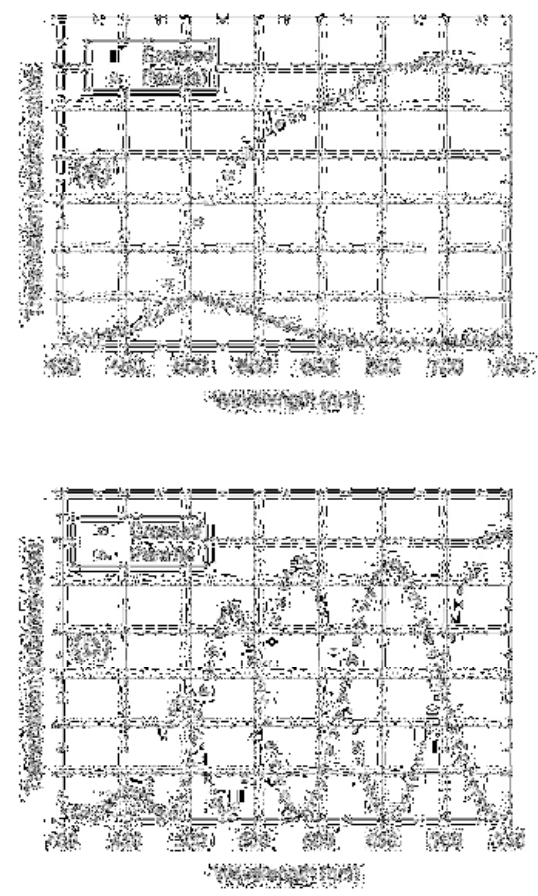

Fig. 2. Transmission through parallel aligned cellophane tape layers inserted between crossed and parallel polarizers oriented at $45^{\circ}$ with respect to the principal axis. (a) 1 layer, (b) 8 layers. 
The second design is a birefringent fil which provides a transmission th approximates a square wave, with flat regio near zero and $100 \%$ transmission [6]. Ag: the filter is placed between two cross polarizers. It consists on four cellophane ta layers oriented at $+45^{\circ}$ with respect to $t$ polarizers, followed by other eight laye oriented at $-15^{\circ}$, and finally other eight ta layers oriented at $+10^{\circ}$. Figure $3(\mathrm{~b})$ shows $t$ simulated transmission together with $t$ experimental data obtained by $t$ spectrophotometer. Again, an excelle agreement is obtained.

\section{Conclusion}

Summarizing, we have demonstrated a ve simple way of building birefringent filte based on the use of cellophane tape laye We first calibrated the birefringence of $t$ tape, and used this information to design the filters. This technique represents an inexpensive way of experience the properties and applications of the polarization of light.

\section{Acknowledgments}

This work was supported by Ministerio de Ciencia y Tecnología from Spain (BFM200306273-C02-02/FISI) and Generalitat Valenciana (GRUPOS03/117).

\section{References}

[1] D. S. Goodman, "Polarization," in Optics Demonstrations with the Overhead Projector, SPIE Press, Washington DC (2000).

[2] C. Gang, "Cellophane Tape Kaleidoscope," in The Chemistry of Polymers, Terrific Science Books, New York (2004).

[3] E. Hecht, Optics, 4th Ed., Addison-Wesley, Reading (1997).

[4] P. Yeh, Optical Waves in Layered Media, Wiley, New York (1988).

[5] P. Velásquez, M. M. Sánchez-López, I. Moreno, D. Puerto and F. Mateos, "Interference birefringent filtres with low cost commercial polymers," Am. J. Phys. 73 (4), 357-361 (2005).

[6] W. J. Carlsen and C. F. Buhrer, "Flat passband birefringent wavelength division multiplexer," Electron. Lett. 23, 106-107 (1987). 